\title{
A Simple Stochastic Process Model for River Environmental Assessment Under Uncertainty
}

\author{
Hidekazu Yoshioka ${ }^{1(\bowtie)}(\mathbb{D})$, Motoh Tsujimura $^{2}$ (D), \\ Kunihiko Hamagami ${ }^{3}$, and Yumi Yoshioka ${ }^{1}$ \\ ${ }^{1}$ Shimane University, Nishikawatsu-cho 1060, Matsue 690-8504, Japan \\ \{yoshih, yyoshioka\}@life.shimane-u.ac.jp \\ ${ }^{2}$ Graduate School of Commerce, Doshisha University, \\ Karasuma-Higashi-iru, Imadegawa-dori, Kyoto 602-8580, Japan \\ mtsujimu@mail.doshisha.ac.jp \\ ${ }^{3}$ Faculty of Agriculture, Iwate University, \\ 3-18-8 Ueda, Morioka 020-8550, Japan \\ ham@iwate-u.ac.jp
}

\begin{abstract}
We consider a new simple stochastic single-species population dynamics model for understanding the flow-regulated benthic algae bloom in uncertain river environment: an engineering problem. The population dynamics are subject to regime-switching flow conditions such that the population is effectively removed in a high-flow regime while it is not removed at all in a lowflow regime. A focus in this paper is robust and mathematically rigorous statistical evaluation of the disutility by the algae bloom under model uncertainty. We show that the evaluation is achieved if the optimality equation derived from a dynamic programming principle is solved, which is a coupled system of nonlinear and non-local degenerate elliptic equations having a possibly discontinuous coefficient. We show that the system is solvable in continuous viscosity and asymptotic senses. We also show that its solutions can be approximated numerically by a convergent finite difference scheme with a demonstrative example.
\end{abstract}

Keywords: Regime-switching stochastic process $\cdot$ Model uncertainty $\cdot$ Environmental problem $\cdot$ Viscosity solution

\section{Introduction}

This paper focuses on a population dynamics modeling of nuisance benthic algae on riverbed under uncertain environment: a common environmental problem encountered in many rivers where human regulates the flow regimes [1]. Blooms of nuisance benthic algae and macrophytes, such as Cladophora glomerata and Egeria densa, in inland water bodies are seriously affecting aquatic ecosystems [2, 3]. Such environmental problems are especially severe in dam-downstream rivers where the flow regimes are often regulated to be low, with which the nuisance algae can dominate the others $[4,5]$. 
It has been found that the benthic algae are effectively removed when they are exposed to a sufficiently high flow discharge containing sediment particles [6]. Supplying sediment into a river environment can be achieved through transporting earth and soils from the other sites, as recently considered in Yoshioka et al. [7] focusing on a case study in Japan with a high-dimensional stochastic control model.

Assume that we could find a way to supply the sediment into a river environment where the nuisance algae are blooming. Then, a central issue is to what extent the algae bloom can be suppressed in the given environment. Hydrological studies imply that river flows are inherently stochastic and can be effectively described using a Markovchain [8]. In the simplest case, we can classify river flow regimes into the two regimes: a high-flow regime where the nuisance algae can be effectively removed from the riverbed and a low-flow regime where they are not removed from the riverbed at all. In this view, the algae population dynamics can be considered as a stochastic dynamical system subject to a two-state regime-switching noise. To the best of our knowledge, such an attempt has been least explored despite its high engineering importance.

We approach this issue both mathematically and numerically. We formulate the algae population dynamics as a system of piecewise-deterministic system subject to a Markovian regime-switching noise [9] representing a dynamic river flow having highand low-flow regimes. This is a system of stochastic differential equations (SDEs, Øksendal and Sulem [10]) based on a logistic model subject to the detachment during the high-flow regime [7] but with a simplification for better tractability. The model incorporates our own experimental evidence that a sudden detachment of the algae occurs when the flow regime switches from the high-flow to the low-flow. This finding introduces a non-locality into the model.

Our focus is not only on the population dynamics themselves, but also on statistical evaluation of the dynamics that can also be important in engineering applications. Namely, another focus is the evaluation of statistical indices such as a disutility caused by the population, which are given by conditional expectations of quantities related to the population. Unfortunately, it is usually difficult to accurately identify model parameters in the natural environment due to technical difficulties and poor data availability. In such cases, we must operate a model under the assumption that it is incomplete and thus uncertain (or equivalently, ambiguous). We overcome this issue by employing the concept of multiplier robust control [11], which allows us to analyze SDEs having uncertainty and further to statistically evaluate their dynamics in a worstcase robust manner. This methodology originates from economics and has been employed in finance [12] and insurance [13], but less frequently in environment and ecology [14]. With this formulation, we demonstrate that the stochastic dynamics having model uncertainty can be handled mathematically rigorously as well as efficiently.

We show that the robust evaluation of a statistical index related to the population dynamics ultimately reduces to solving a system of non-linear and non-local degenerate elliptic equations: the optimality equation having a possibly discontinuous source term. This is the governing equation of the statistical index under the worst-case. Our goal is therefore to solve the equation in some way. We show that solutions to the optimality equation are characterized in a viscosity sense [15], and that it admits a continuous viscosity solution by a comparison theorem [16]. We present an analytical asymptotic 
estimate of the solution as well. We finally provide a demonstrative computational example with a convergent finite difference scheme [5, 14] to show the validity of the asymptotic estimate and to deeper comprehend the behavior of the model.

\section{Mathematical Model}

\subsection{System Dynamics}

Let $\left(\alpha_{t}\right)_{t \geq 0}$ be a càdlàg two-state continuous-time Markov chain having a low-flow regime $(i=0)$ and a high-flow regime $(i=1)$. The switching rate from the regimes 0 to 1 (resp., 1 to 0 ) is a positive constant $v_{01}>0$ (resp., $v_{10}>0$ ). We assume that the regime switching occurs with the prescribed switching rates and some Poisson processes. We thus describe the SDE governing temporal evolution of $\left(\alpha_{t}\right)_{t \geq 0}$ as

$$
\mathrm{d} \alpha_{t}=\chi_{\left\{\alpha_{t-}=0\right\}} \mathrm{d} N_{t}^{(01)}-\chi_{\left\{\alpha_{t-}=1\right\}} \mathrm{d} N_{t}^{(10)} \text { for } t \geq 0, \alpha_{0-} \in\{0,1\},
$$

where $\chi_{S}$ is the indicator function such that $\chi_{S}=1$ if $S$ is true and $\chi_{S}=0$ otherwise, $\alpha_{t-}=\lim _{s \rightarrow+0} \alpha_{t-s}$ and the same representation applies to the other processes, $\left(N_{t}^{(01)}\right)_{t \geq 0}$ and $\left(N_{t}^{(10)}\right)_{t \geq 0}$ are mutually-independent standard Poisson processes with the jumping rates $v_{01}$ and $v_{10}$, respectively. The switching times from the regimes 0 to 1 (resp., regimes 1 to 0 ) are represented by a strictly increasing sequence $\left(\tau_{k}^{01}\right)_{k \in \mathbb{N}}$ (resp., $\left.\left(\tau_{k}^{10}\right)_{k \in \mathbb{N}}\right)$. We assume a.s. $\tau_{k}^{10} \neq \tau_{l}^{01}(k, l \in \mathbb{N})$ without loss of generality.

The algae population is represented by a continuous-time variable $\left(X_{t}\right)_{t \geq 0}$, which is assumed to be governed by a generalized logistic model having regime-switching coefficients. The difference between the regimes 0 and 1 in the context of the population dynamics is that the algae detachment occurs only at the regime 1 (high-flow regime). We normalize the population $X_{t}$ to be valued in $[0,1]$. We set the SDE of $\left(X_{t}\right)_{t \geq 0}$ as

$$
\mathrm{d} X_{t}=\left(\mu\left(1-X_{t-}^{\theta}\right)-\chi_{\left\{\alpha_{t-}=1\right\}} D\left(X_{t-}\right)\right) X_{t-} \mathrm{d} t \text { for } t \geq 0, X_{0-} \in[0,1]
$$

where $\theta \geq 1$ is the shape parameter, $\mu>0$ is the specific growth rate and $D:[0,1] \rightarrow$ $[0,+\infty)$ is the detachment rate that is assumed to be non-negative and Lipschitz continuous in $[0,1]$ and $D(1)>0$. One of our own important experimental findings is that a sudden algae detachment occurs at the initiation of each high-flow event when there is an enough sediment supply. From the standpoint of the present model, this imposes the additional state dynamics at the switching times $X_{\tau_{k}^{01}}=\left(1-z_{k}\right) X_{\tau_{k}^{01}-}$ for $k \in \mathbb{N}$, where $\left(z_{k}\right)_{k \in \mathbb{N}}$ is a sequence of i.i.d. stochastic variables representing the sudden detachment of the algae population. We assume that they have the common compact range $Z \subset(0,1)$, meaning that the sudden decrease of the population does not lead to its immediate extinction, which is consistent with the empirical finding [5]. The sequence $\left(z_{k}\right)_{k \in \mathbb{N}}$ is assumed to be independent from $N_{t}^{(01)}$ and $N_{t}^{(10)}$. The probability density function generating $\left(z_{k}\right)_{k \in \mathbb{N}}$ is denoted as $g \geq 0$ with $\int_{Z} g(z) \mathrm{d} z=1$. 
The stochastic nature of the detachment has been justified from our experimental results. We found that the amount of sudden detachment is different among the experimental runs even under the same experimental setting (sediment supply and water flow). This is considered due to inherently probabilistic nature of the sediment supply and microscopic difference of biological conditions (length, density, rock shape, etc.,) of the algae population. The same would be true in real river environment.

The natural filtration generated by $\left(N_{t}^{(01)}\right)_{t \geq 0},\left(N_{t}^{(10)}\right)_{t \geq 0}$, and $\left(z_{k}\right)_{k \in \mathbb{N}}$ at time $t$ are denoted as $F_{t}$. Set $F=\left(F_{t}\right)_{t \geq 0}$. Consequently, the coupled stochastic system dynamics to be observed are given as follows $(k \in \mathbb{N})$ :

$$
\begin{aligned}
& \mathrm{d} \alpha_{t}=\chi_{\left\{\alpha_{t-}=0\right\}} \mathrm{d} N_{t}^{(01)}-\chi_{\left\{\alpha_{t-}=1\right\}} \mathrm{d} N_{t}^{(10)}, t \geq 0 \\
& \mathrm{~d} X_{t}=\left(\mu\left(1-X_{t-}^{\theta}\right)-\chi_{\left\{\alpha_{t-}=1\right\}} D\left(X_{t-}\right)\right) X_{t-} \mathrm{d} t, t \geq 0, t \neq \tau_{k}^{01},\left(\alpha_{0-}, X_{0-}\right) \in\{0,1\} \times[0,1] . \\
& X_{\tau_{k}^{01}}=\left(1-z_{k}\right) X_{\tau_{k}^{01-}}, k \in \mathbb{N}
\end{aligned}
$$

\subsection{Performance Index}

A performance index to statistically evaluate the stochastic population dynamics (3) is formulated. To simplify the problem as much as possible, we consider the following conditional expectation evaluating the disutility caused by the algae population:

$$
\Phi(i, x)=\mathbb{E}^{i, x}\left[\int_{0}^{+\infty} f\left(X_{s}\right) e^{-\delta s} \mathrm{~d} s\right],(i, x) \in\{0,1\} \times[0,1],
$$

where $\mathbb{E}^{i, x}$ is the conditional expectation with $\left(\alpha_{0-}, X_{0_{-}}\right)=(i, x), f \geq 0$ is a bounded upper-semicontinuous function on $[0,1]$ having at most a finite number of discontinuous points in $(0,1)$. Such a discontinuity naturally arises if there is a threshold above which the algae bloom would severely affect the water environment. The performance index (4) is the mean value of an infinite-horizon discounted disutility. We write $\Phi(i, x)=\Phi_{i}(x)$ if there will be no confusion.

The dynamic programming principle [10] formally leads to the governing equation of $\Phi_{i}$ as the system of linear degenerate elliptic equations

$$
\begin{aligned}
& \delta \Phi_{0}-\mu\left(1-x^{\theta}\right) x \frac{\mathrm{d} \Phi_{0}}{\mathrm{~d} x}+v_{01} \int_{Z} \Delta_{01} \Phi g(z) \mathrm{d} z-f=0, x \in[0,1] \\
& \delta \Phi_{1}-\left(\mu\left(1-x^{\theta}\right)-D(x)\right) x \frac{\mathrm{d} \Phi_{1}}{\mathrm{~d} x}+v_{10} \Delta_{10} \Phi-f=0, x \in[0,1]
\end{aligned}
$$

with

$$
\Delta_{01} \Phi(x, z)=\Phi_{0}(x)-\Phi_{1}(x(1-z)) \text { and } \Delta_{10} \Phi(x)=\Phi_{1}(x)-\Phi_{0}(x)
$$




\subsection{A Model with Uncertainty}

We consider an uncertain counterpart, where the model uncertainty is assumed due to distortions of $\left(N_{t}^{(01)}\right)_{t \geq 0},\left(N_{t}^{(10)}\right)_{t \geq 0}$, and $g$. Namely, we focus on hydrological uncertainties. Our formulation is based on the models with uncertain jump processes $[11,12]$ with our notations of the parameters and variables. Let $\mathbb{P}$ be the current probability measure and $\mathbb{Q}$ be the probability measure under the distortion. Set $\left(\phi_{t}^{i}(\cdot)\right)_{t \geq 0}(i=0,1)$ as positive and strictly bounded $F$-predictable random fields representing model uncertainty. There is no uncertainty when $\phi_{t}^{0}=\phi_{t}^{1}=1(t \geq 0)$. Recall that there is no jump of $X_{t}$ at each $\tau_{k}^{10}$. The new and old probability measures are characterized by the Radon-Nikodym derivative $\frac{\mathrm{d} \mathbb{Q}}{\mathrm{d} \mathbb{P}}=\Lambda_{t}^{0} \Lambda_{t}^{1}$, where

$$
\begin{gathered}
\Lambda_{t}^{0}=\exp \left(\int_{Z}\left(\int_{0}^{t}\left(1-\phi_{s}^{0}(z)\right) v_{01} \mathrm{~d} s+\int_{0}^{t} \ln \phi_{s}^{0}(z) \mathrm{d} N_{s}^{(01)}\right) g(z) \mathrm{d} z\right), \\
\Lambda_{t}^{1}=\exp \left(\int_{0}^{t}\left(1-\phi_{s}^{1}\right) v_{01} \mathrm{~d} s+\int_{0}^{t} \ln \phi_{s}^{1} \mathrm{~d} N_{s}^{(10)}\right)
\end{gathered}
$$

under the assumption that the right-hand sides are a.s. bounded for $t \geq 0$. We thus assume that $\Lambda_{t}^{0}$ and $\Lambda_{t}^{1}$ are positive and a.s. bounded for $t \geq 0$. This is true if the processes $\left(\phi_{t}^{i}(\cdot)\right)_{t \geq 0}(i=0,1)$ are strictly positive and uniformly bounded. Notice that $\phi_{t}^{1}$ is actually a function only of time $t$, but is represented as $\phi_{t}^{1}(\cdot)$ here for convenience. Formally, at time $t$, the jump intensity of $N_{t}^{(01)}$ (resp., $N_{t}^{(10)}$ ) is modified from $v_{01}$ to $v_{01} \int_{Z} \phi_{t}^{0}(z) g(z) \mathrm{d} z$ (resp., $v_{10}$ to $v_{10} \phi_{t}^{1}(z)$ ) and the probability density function $g=g(z)$ to $C \phi_{t}^{0}(z) g(z)$ with a constant $C>0$. The support of $\phi_{t}^{0}(z) g(z)$ is still $Z$. We impose the following normalization, so that the modified $g$, which is written as $g^{*}$, is truly a probability density function:

$$
g^{*}(z)=\frac{\phi_{t}^{0}(z) g(z)}{\int_{Z} \phi_{t}^{0}(z) g(z) \mathrm{d} z}, t \geq 0 .
$$

Under the new probability measure $\mathbb{Q}$, the processes $N_{t}^{(01)}$ and $N_{t}^{(10)}$ are formally replaced by the new Poisson processes $\bar{N}_{t}^{(01)}$ and $\bar{N}_{t}^{(10)}$ having the jump intensities $v_{01} \int_{Z} \phi_{t}^{0}(z) g(z) \mathrm{d} z$ and $v_{10} \phi_{t}^{1}$, respectively, both of which are assumed to be strictly positive and bounded. Now, the transformed system dynamics are $(k \in \mathbb{N})$ :

$$
\begin{aligned}
& \mathrm{d} \alpha_{t}=\chi_{\left\{\alpha_{t-}=0\right\}} \mathrm{d} \bar{N}_{t}^{(01)}-\chi_{\left\{\alpha_{t-}=1\right\}} \mathrm{d} \bar{N}_{t}^{(10)}, t \geq 0 \\
& \mathrm{~d} X_{t}=\left(\mu\left(1-X_{t-}^{\theta}\right)-\chi_{\left\{\alpha_{-}=1\right\}} D\left(X_{t-}\right)\right) X_{t-} \mathrm{d} t, t \geq 0, t \neq \tau_{k}^{01},\left(\alpha_{0-}, X_{0-}\right) \in\{0,1\} \times[0,1] \\
& X_{\tau_{k}^{01}}=\left(1-z_{k}\right) X_{\tau_{k}^{01}-}, k \in \mathbb{N}
\end{aligned}
$$


The performance index $J$ is then extended to a worst-case uncertain counterpart, with which the observer can evaluate the disutility subject to an entropic penalization for the deviations between the true and distorted models:

$$
\Phi(i, x)=\sup _{\left(\phi_{t}^{01}(\cdot), \phi_{t}^{10}\right)_{t \geq 0}} J\left(i, x ; \phi^{01}, \phi^{10}\right),(i, x) \in\{0,1\} \times[0,1]
$$

with

$$
\begin{gathered}
J\left(i, x ; \phi^{01}, \phi^{10}\right)=\mathbb{E}^{i, x}\left[\int_{0}^{+\infty} f\left(X_{s}\right) e^{-\delta s} \mathrm{~d} s-\left(\frac{v_{01}}{\psi_{0}} I_{0}+\frac{v_{10}}{\psi_{1}} I_{1}\right)\right], \\
I_{0}=\int_{0}^{+\infty} e^{-\delta s} \int_{Z}\left(\phi_{s}^{0}(z) \ln \phi_{s}^{0}(z)-\phi_{s}^{0}(z)+1\right) g(z) \mathrm{d} z \mathrm{~d} s, \\
I_{1}=\int_{0}^{+\infty} e^{-\delta s}\left(\phi_{s}^{1} \ln \phi_{s}^{1}-\phi_{s}^{1}+1\right) \mathrm{d} s,
\end{gathered}
$$

where $\psi_{0}>0$ and $\psi_{1}>0$ are the uncertainty-aversion parameters serving as penalization parameters to constrain the allowable difference between the true and distorted models. The integrands of (14) and (15) are (discounted) relative entropy between the models with respect to $\phi_{t}^{0}$ and $\phi_{t}^{1}$, respectively. Each uncertainty-aversion parameter modulates the corresponding relative entropy in the way that a larger parameter allows for a larger deviation between the true and distorted models, and vice versa. This formulation reduces to the model without uncertainty as $\psi_{0}, \psi_{1} \rightarrow+0$. In this way, the observer can flexibly presume the potential uncertainty.

The dynamic programming principle [10] leads to the governing equation of the value function $\Phi_{i}$ subject to model uncertainty as the coupled system of non-linear and non-local degenerate elliptic equations in $[0,1]$ :

$$
\begin{aligned}
& \delta \Phi_{0}-\mu\left(1-x^{\theta}\right) x \frac{\mathrm{d} \Phi_{0}}{\mathrm{~d} x}-f \\
& +v_{01} \inf _{\phi^{0}(\cdot)>0}\left\{\int_{Z}\left(\phi^{0}(z) \Delta_{01} \Phi+\frac{1}{\psi_{0}}\left(\phi^{0}(z) \ln \phi^{0}(z)-\phi^{0}(z)+1\right)\right) g(z) \mathrm{d} z\right\}=0
\end{aligned}
$$

and

$$
\begin{aligned}
& \delta \Phi_{1}-\left(\mu\left(1-x^{\theta}\right)-D(x)\right) x \frac{\mathrm{d} \Phi_{1}}{\mathrm{~d} x}-f \\
& +v_{10} \inf _{\phi^{1}>0}\left\{\phi^{1} \Delta_{10} \Phi+\frac{1}{\psi_{1}}\left(\phi^{1} \ln \phi^{1}-\phi^{1}+1\right)\right\}=0 .
\end{aligned}
$$


The minimizations in (16) and (17) are achieved by the worst-case uncertainties as

$$
\phi^{0 *}(x, z)=\exp \left(-\psi_{0} \Delta_{01} \Phi(x, z)\right) \text { and } \phi^{1 *}(x)=\exp \left(-\psi_{1} \Delta_{10} \Phi(x)\right)
$$

Substituting (18) into (16) and (17) simplifies them to

$$
\delta \Phi_{0}-\mu\left(1-x^{\theta}\right) x \frac{\mathrm{d} \Phi_{0}}{\mathrm{~d} x}+\frac{v_{01}}{\psi_{0}} \int_{Z}\left(1-\exp \left(-\psi_{0} \Delta_{01} \Phi\right)\right) g(z) \mathrm{d} z-f=0,
$$

and

$$
\delta \Phi_{1}-\left(\mu\left(1-x^{\theta}\right)-D(x)\right) x \frac{\mathrm{d} \Phi_{1}}{\mathrm{~d} x}+\frac{v_{10}}{\psi_{1}}\left(1-\exp \left(-\psi_{1} \Delta_{10} \Phi\right)\right)-f=0 .
$$

We formally derive (5)-(6) under $\psi_{0}, \psi_{1} \rightarrow+0$ as expected. The quantities in (18) represent the worst-case uncertainties conditioned on the current observations of $\alpha$ and $X$. Furthermore, by (10), the probability density function $g^{*}$ under the distortion is

$$
g^{*}(x, z)=\frac{\exp \left(-\psi_{0} \Delta_{01} \Phi\right) g(z)}{\int_{Z} \exp \left(-\psi_{0} \Delta_{01} \Phi\right) g(z) \mathrm{d} z}
$$

with an abuse of notations. The worst-case jump intensities from the regimes 0 to 1 and 1 to 0 , which are represented as $v_{01}^{*}=v_{01}^{*}(x)$ and $v^{*}=v_{10}^{*}(x)$, are given by

$$
v_{01}^{*}(x)=v_{01} \int_{Z} \exp \left(-\psi_{0} \Delta_{01} \Phi\right) g(z) \mathrm{d} z \text { and } v_{10}^{*}(x)=v_{10} \exp \left(-\psi_{1} \Delta_{10} \Phi(x)\right) .
$$

\section{Mathematical Analysis}

\subsection{Viscosity Solution}

Boundedness and continuity of the value function are analyzed. We firstly prove unique solvability of the system (11).

Proposition 1. The system (11) admits a unique strong solution such that $0 \leq X_{t} \leq 1$ $(t \geq 0)$.

Proof: A similar contradiction argument to that in the proof of Theorem 2.2 of Lungu and Øksendal [17] applies in our case. We can get unique existence of the system having coefficients extended to be Lipschitz continuous over $\mathbb{R}$, by Theorem 2.1 of Yin and Zhu [9]. With a contradiction argument [17], we obtain that the strong solution to this modified problem is bounded in $[0,1]$.

By Proposition 1, we get a continuity result of the value function, with which an appropriate definition of viscosity solutions to the optimality equation (19)-(20) is found. 
Proposition 2. Assume that $f$ is Hölder continuous in $[0,1]$. Then, we get $\Phi_{i} \in C[0,1]$, $(i=0,1)$.

Proof: Combine the strong solution property and the boundedness result in Proposition 1 with the Hölder continuity of $f$.

Now, we define viscosity solutions. Set the space of upper-semicontinuous (resp., lower-semicontinuous) functions in $[0,1]$ as $\operatorname{USC}[0,1]$ (resp., $\operatorname{LSC}[0,1]$ ).

Definition 1. A pair $\Psi_{0}, \Psi_{1} \in U S C[0,1]$ is a viscosity sub-solution iffor all $x_{0} \in[0,1]$ and $i_{0}=0,1$, and for all $\varphi_{0}, \varphi_{1} \in C^{1}[0,1]$ s.t. $\varphi_{i}-\Psi_{i}$ is locally minimized at $x=x_{0}$ and $i=i_{0}$ with $\varphi_{i_{0}}\left(x_{0}\right)=\Psi_{i_{0}}\left(x_{0}\right)$, the following hold ((23) for $i_{0}=0$, (24) for $\left.i_{0}=1\right)$ :

$$
\begin{aligned}
& \delta \Psi_{0}-\mu\left(1-x^{\theta}\right) x \frac{\mathrm{d} \varphi_{0}}{\mathrm{~d} x}+\frac{v_{01}}{\psi_{0}} \int_{Z}\left(1-\exp \left(-\psi_{0} \Delta_{01} \Psi\right)\right) g(z) \mathrm{d} z-f^{*}(x) \leq 0, x=x_{0}, \\
& \delta \Psi_{1}-\left(\mu\left(1-x^{\theta}\right)-D(x)\right) x \frac{\mathrm{d} \varphi_{1}}{\mathrm{~d} x}+\frac{v_{10}}{\psi_{1}}\left(1-\exp \left(-\psi_{1} \Delta_{10} \Psi\right)\right)-f^{*}(x) \leq 0, x=x_{0} .
\end{aligned}
$$

A pair $\Psi_{0}, \Psi_{1} \in L S C[0,1]$ is a viscosity super-solution if for all $x_{0} \in[0,1]$ and $i_{0}=0,1$, and for all $\varphi_{0}, \varphi_{1} \in C^{1}[0,1]$ s.t. $\varphi_{i}-\Psi_{i}$ is locally maximized at $x=x_{0}$ and $i=i_{0}$ with $\varphi_{i_{0}}\left(x_{0}\right)=\Psi_{i_{0}}\left(x_{0}\right)$, the following hold ((25) for $i_{0}=0$, (26) for $\left.i_{0}=1\right)$ :

$$
\begin{gathered}
\delta \Psi_{0}-\mu\left(1-x^{\theta}\right) x \frac{\mathrm{d} \varphi_{0}}{\mathrm{~d} x}+\frac{v_{01}}{\psi_{0}} \int_{Z}\left(1-\exp \left(-\psi_{0} \Delta_{01} \Psi\right)\right) g(z) \mathrm{d} z-f_{*}(x) \geq 0, x=x_{0} \\
\delta \Psi_{1}-\left(\mu\left(1-x^{\theta}\right)-D(x)\right) x \frac{\mathrm{d} \varphi_{1}}{\mathrm{~d} x}+\frac{v_{10}}{\psi_{1}}\left(1-\exp \left(-\psi_{1} \Delta_{10} \Psi\right)\right)-f_{*}(x) \geq 0, x=x_{0} .
\end{gathered}
$$

A pair $\Psi_{0}, \Psi_{1} \in C[0,1]$ is a viscosity solution if it is a viscosity sub-solution as well as a viscosity super-solution.

Proposition 3. Assume that $f$ is Hölder continuous in [0,1]. Then, the value function is a viscosity solution.

Proof: Apply the Dynkin's formula and the dominated convergence theorem.

Proposition 4. For any viscosity sub-solution u and a viscosity super-solution $v, v \geq u$ in $\Omega$. Moreover, the optimality equation (19)-(20) admits at most one viscosity solution.

Proof: Apply a contradiction argument with the help of the monotonicity of the nonlinear terms. [Proof of Proposition 3.3 in 14] and [Proof of Theorem 11.4 in 16].

Notice that the uniqueness result in Proposition 4 holds true for both continuous and discontinuous $f$. A consequence of Propositions 3 and 4 is the next theorem. 
Theorem 1. Assume that $f$ is Hölder continuous in $[0,1]$. Then, the value function is the unique viscosity solution to the optimality equation. (19)-(20).

\subsection{Asymptotic Solution}

The optimality equation is uniquely solvable, but its exact solution cannot be found analytically. Instead, we construct an asymptotic solution that close to the solution for small $x$. The asymptotic solution thus applies to the situation where the population is sufficiently small, which would be encountered during a high-flow regime. The next proposition can be checked by a direct calculation. The asymptotic worst-case uncertainties can also be calculated using this proposition.

Proposition 5. Assume $\Phi_{i} \in C^{2}[0, \varepsilon)(i=0,1)$ with $0<\varepsilon<1$. Assume that for small $0<x<\varepsilon, f(x)=x^{m}+O\left(x^{m+v}\right)$ and $D(x)=d+O\left(x^{v}\right), d \geq 0, m, v>0, \delta>0$ such that

$$
A=\left(\delta-m \mu+v_{01}\right)\left(\delta-m(\mu-d)+v_{01}\right)-v_{01} v_{10} \int_{Z}(1-z)^{m} g(z) \mathrm{d} z>0
$$

Then, we have the following asymptotic expansions for small $x>0$ :

$$
\Phi_{i}(x)=C_{i} x^{m}+\text { higher-order terms of } x(i=0,1),
$$

with the positive constants

$$
C_{0}=\frac{\delta-m(\mu-d)+v_{01} \int_{Z}(1-z)^{m} g(z) \mathrm{d} z+v_{10}}{A} \text { and } C_{1}=\frac{\delta-m \mu+v_{01}+v_{10}}{A} .
$$

\section{Numerical Computation}

We numerically discretize the optimality equation (19)-(20) because it is not analytically solvable and the asymptotic solution presented in the previous section can be utilized only under limited conditions. The employed numerical method here is the finite difference scheme with the Newton iteration [5], which can handle the decay and first-order differential terms of the degenerate elliptic differential equations. The nonlinear and non-local terms are handled with the interpolation technique of [14]. This scheme is a version of the non-standard finite difference scheme based on local exact solutions to linearized problems [18], with which monotone, stable, and consistent discretization is established. It is thus convergent in a viscosity sense [19]. The nonlocal term is linearized at each iteration step to enhance computational stability. 


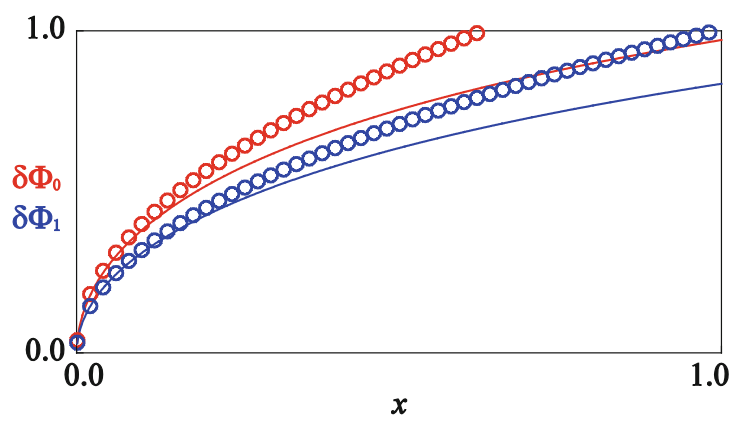

Fig. 1. The computed and asymptotic $\delta \Phi_{0}(x)$ (red) and $\delta \Phi_{1}(x)$ (blue) with $\psi=1$. Line: computed result, Circle: asymptotic result. (Color figure online)

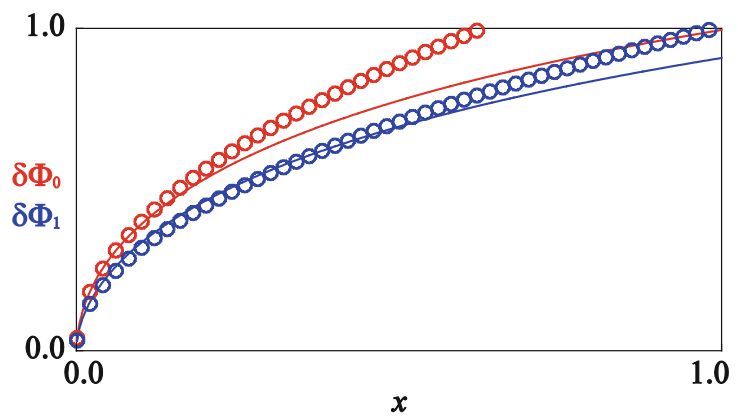

Fig. 2. The computed and asymptotic $\delta \Phi_{0}(x)$ (red) and $\delta \Phi_{1}(x)$ (blue) with $\psi=20$. Line: computed result, Circle: asymptotic result. (Color figure online)

The computational condition here is specified as follows. For the sake of simplicity, we set $\psi_{0}=\psi_{1}=\psi>0$. In addition, set $D(x)=d x$ and $f(x)=x^{m}$ with $d, m>0$. The computational domain is discretized into 1,000 cells with 1,001 vertices, and the range $Z$ of the uncertainty into 500 cells. The following parameter values are used in the computation: $\quad m=0.5, \delta=2, \mu=0.5, \theta=1, d=1, v_{01}=0.1, v_{10}=1.0, g(z)=$ $3 \chi_{\{1 / 3 \leq z \leq 2 / 3\}}$, and $\psi=1$ or 20 . The threshold to terminate the iteration is

$$
\max _{i=0,1} \frac{\left|\Phi_{i}^{(n+1)}-\Phi_{i}^{(n)}\right|}{\max \left\{1, \Phi_{i}^{(n)}\right\}}<10^{-12}
$$

at all the computational vertices, where the superscript represents the iteration number in the Newton iteration. The numerical solution $\Phi_{i}^{(n+1)}$ with the smallest $n$ satisfying (30) is considered as the numerical solution. Each computation below requires at most 20 to 30 steps, implying satisfactory efficiency of the scheme. 


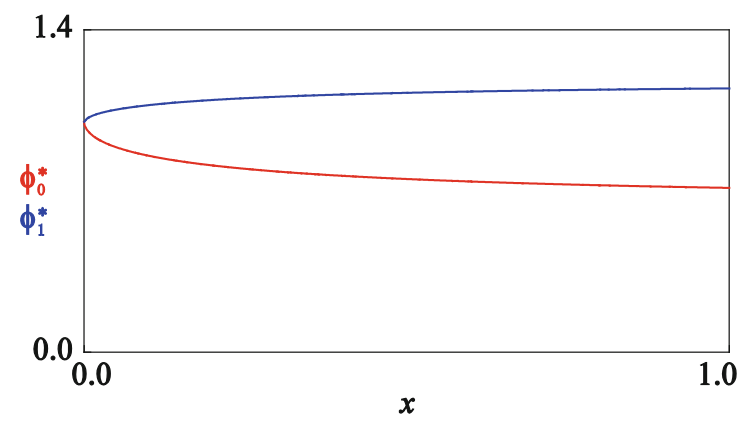

Fig. 3. The computed $\phi_{0}^{*}(x)$ (red) and $\phi_{1}^{*}(x)$ (blue) with $\psi=1$. (Color figure online)

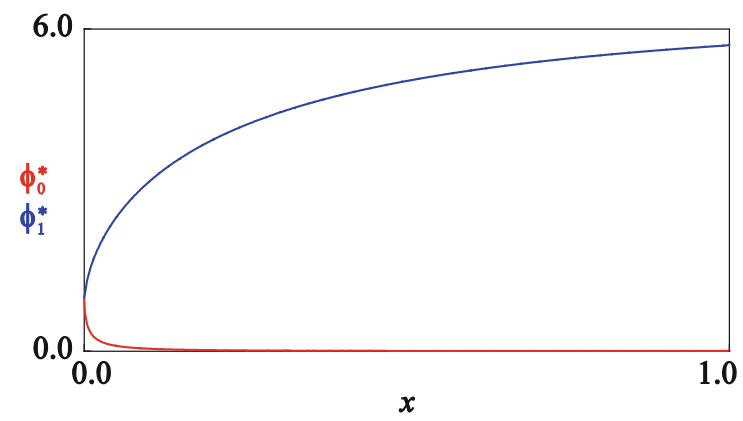

Fig. 4. The computed $\phi_{0}^{*}(x)$ (red) and $\phi_{1}^{*}(x)$ (blue) with $\psi=20$. (Color figure online)

In Figs. 1 and 2, the numerically computed value functions (solid lines) are compared with the asymptotic results for $\psi=1$ and $\psi=20$, suggesting their good agreement especially for small $x$. We can see that the disutility assessed by the more uncertainty-averse observer (having a larger $\psi$ ) is evaluated larger. In Figs. 3 and 4, the worst-case uncertainties $\phi_{0}^{*}(x)=\int_{Z} \phi_{0}^{*}(z, x) g(z) \mathrm{d} z$ and $\phi_{1}^{*}(x)$ are compared for $\psi=1$ and $\psi=20$, suggesting the decreasing and increasing nature of the former and latter, respectively. The magnitude of decrease/increase more sharply depends on the population for larger $\psi$. The monotone dependence of $\phi_{i}^{*}$ shows that the observer considers the flood frequency smaller under larger uncertainty-aversion.

Figure 5 shows the worst-case probability density functions $g^{*}(z, x)$ for different values of $x$ when $\psi=20$. The computational results clearly show the distortion of $g$ due to model uncertainty; larger distortion is observed for larger population. The worstcase probability density functions are positively-skewed, implying that the more uncertainty-averse observer specifies thus sudden detachment of the algae population to be smaller. In addition, their skewness significantly depends on the observed population, suggesting that the more skewed prediction is optimal when the population is large: namely, in case of the algae bloom. 


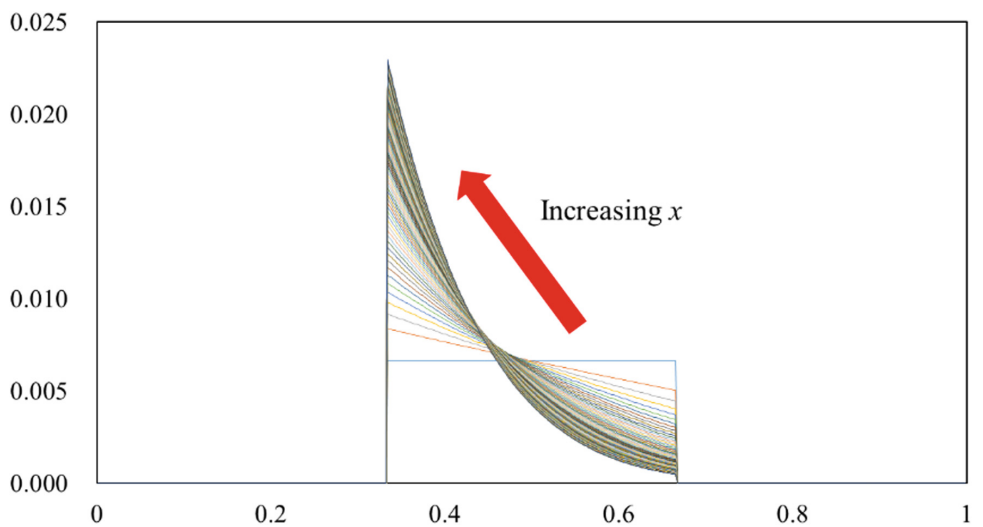

Fig. 5. The computed worst-case probability density functions $g^{*}(z, x)(x=i / 100(i=$ $0,1,2, \ldots 100))$ for $\psi=20$ : vertical $\left(g^{*}\right)$ and horizontal axes $(z)$.

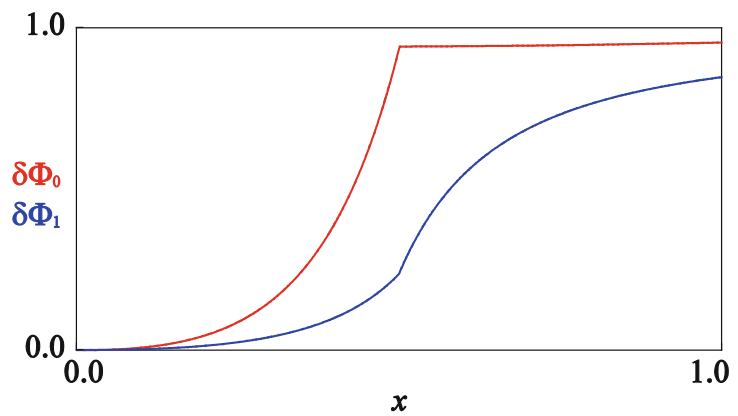

Fig. 6. The computed $\delta \Phi_{0}(x)$ (red) and $\delta \Phi_{1}(x)$ (blue) with a discontinuous f. (Color figure online)

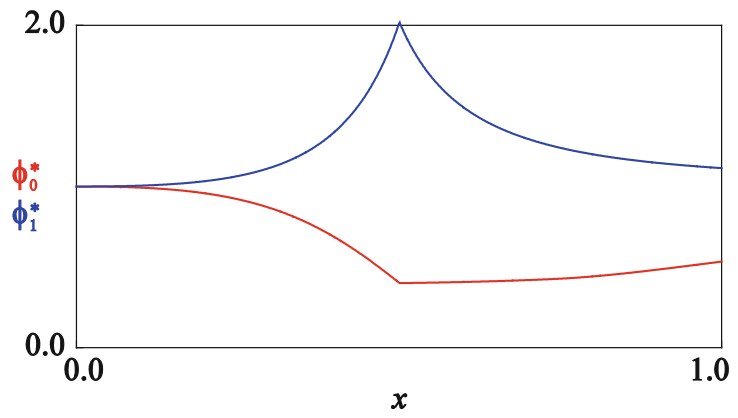

Fig. 7. The computed $\phi_{0}^{*}(x)$ (red) and $\phi_{1}^{*}(x)$ (blue) with a discontinuous f. (Color figure online) 
Finally, we demonstrate that the numerical method applies to discontinuous $f$. We set $f=\chi_{[0.5,1]}$. Figures 6 and 7 show the computed value function and the worst-case uncertainties, suggesting that they have been computed successfully without spurious oscillations. They seem to be not continuously differentiable at the point of discontinuity $x=0.5$. The worst-case uncertainties are now non-monotone, suggesting qualitatively different robust estimations from the previous case with a continuous $f$.

\section{Conclusions}

A simple stochastic model for the algae bloom under uncertain river environment was analyzed both mathematically and numerically. Our framework would provide a mathematically rigorous and computationally feasible framework for resolving the engineering problem. We believe that the framework can also contribute to achieving Sustainable Development Goals (SDGs) related to water environmental and ecological issues.

A future topic would be computing the probability density functions and analyzing stability of the population dynamics. Such analysis can be carried out using the derived or computed worst-case uncertainties as an input. Another topic is a partial observation modeling based on the presented model. For example, one can often directly measure water flows relatively easily (using machines), but only indirectly the algae population.

Acknowledgements. JSPS Research Grant $18 \mathrm{~K} 01714$ and 19H03073, Kurita Water and Environment Foundation Grant No. 19B018, a grant for ecological survey of a life history of the landlocked ayu Plecoglossus altivelis altivelis from the Ministry of Land, Infrastructure, Transport and Tourism of Japan, and a research grant for young researchers in Shimane University support this research.

\section{References}

1. Carey, M.P., Sethi, S.A., Larsen, S.J., Rich, C.F.: A primer on potential impacts, management priorities, and future directions for Elodea spp. in high latitude systems: learning from the Alaskan experience. Hydrobiologia 777(1), 1-19 (2016)

2. Gladyshev, M.I., Gubelit, Y.I.: Green tides: New consequences of the eutrophication of natural waters (Invited Review). Contemp. Probl. Ecol. 12(2), 109-125 (2019)

3. Verhofstad, M.J., Bakker, E.S.: Classifying nuisance submerged vegetation depending on ecosystem services. Limnology 20(1), 55-68 (2019)

4. Yoshioka, H., Yaegashi, Y.: Robust stochastic control modeling of dam discharge to suppress overgrowth of downstream harmful algae. Appl. Stochast. Models Bus. Ind. 34(3), 338-354 (2018)

5. Yoshioka, H.: A simplified stochastic optimization model for logistic dynamics with controldependent carrying capacity. J. Biol. Dyn. 13(1), 148-176 (2019)

6. Hoyle, J.T., Kilroy, C., Hicks, D.M., Brown, L.: The influence of sediment mobility and channel geomorphology on periphyton abundance. Freshw. Biol. 62(2), 258-273 (2017) 
7. Yoshioka, H., Yaegashi, Y., Yoshioka, Y., Hamagami, K.: Hamilton-Jacobi-Bellman quasivariational inequality arising in an environmental problem and its numerical discretization. Comput. Math Appl. 77(8), 2182-2206 (2019)

8. Turner, S.W.D., Galelli, S.: Regime-shifting streamflow processes: Implications for water supply reservoir operations. Water Resour. Res. 52(5), 3984-4002 (2016)

9. Yin, G.G., Zhu, C.: Hybrid Switching Diffusions: Properties and Applications. Springer, New York (2009). https://doi.org/10.1007/978-1-4419-1105-6

10. Øksendal, B., Sulem, A.: Applied Stochastic Control of Jump Diffusions. Springer, Cham (2019)

11. Hansen, L., Sargent, T.J.: Robust control and model uncertainty. Am. Econ. Rev. 91(2), 60$66(2001)$

12. Cartea, A., Jaimungal, S., Qin, Z.: Model uncertainty in commodity markets. SIAM J. Financ. Math. 7(1), 1-33 (2016)

13. Zeng, Y., Li, D., Gu, A.: Robust equilibrium reinsurance-investment strategy for a meanvariance insurer in a model with jumps. Insur. Math. Econ. 66, 138-152 (2016)

14. Yoshioka, H., Tsujimura, M.: A model problem of stochastic optimal control subject to ambiguous jump intensity. In: The 23rd Annual International Real Options Conference London, UK (2019).http://www.realoptions.org/openconf2019/data/papers/370.pdf

15. Crandall, M.G., Ishii, H., Lions, P.L.: User's guide to viscosity solutions of second order partial differential equations. Bull. Am. Math. Soc. 27(1), 1-67 (1992)

16. Calder, J.: Lecture Notes on Viscosity Solutions. University of Minnesota (2018). http:// www-users.math.umn.edu/ jwcalder/viscosity_solutions.pdf. Accessed 22 Dec 2019

17. Lungu, E.M., Øksendal, B.: Optimal harvesting from a population in a stochastic crowded environment. Math. Biosci. 145(1), 47-75 (1997)

18. Mbroh, N.A., Munyakazi, J.B.: A fitted operator finite difference method of lines for singularly perturbed parabolic convection-diffusion problems. Math. Comput. Simul. 165, 156-171 (2019)

19. Barles, G., Souganidis, P.E.: Convergence of approximation schemes for fully nonlinear second order equations. Asymptotic Anal. 4(3), 271-283 (1991) 